\title{
In Vivo Measurement of the Affinity and Density of Metabotropic Glutamate Receptor Subtype 1 in Rat Brain Using ${ }^{18}$ F-FITM in Small-Animal PET
}

\author{
Tomoteru Yamasaki ${ }^{1,2}$, Masayui Fujinaga ${ }^{1}$, Kazunori Kawamura ${ }^{1}$, Joji Yui ${ }^{1}$, Akiko Hatori ${ }^{1}$, Tomoyuki Ohya ${ }^{1}$, Lin Xie ${ }^{1}$, \\ Hidekatsu Wakizaka ${ }^{1}$, Yuichiro Yoshida ${ }^{3}$, Toshimitsu Fukumura ${ }^{1}$, and Ming-Rong Zhang ${ }^{1}$ \\ ${ }^{1}$ Molecular Imaging Center, National Institute of Radiological Sciences, Chiba, Japan; ${ }^{2}$ Graduate School of Pharmaceutical \\ Sciences, Tohoku University, Sendai, Japan; and ${ }^{3}$ SHI Accelerator Service Co. Ltd., Tokyo, Japan
}

Metabotropic glutamate receptor subtype 1 (mGluR1) is a crucial molecular target in the central nervous system disorders. $4-{ }^{18} \mathrm{~F}-$ fluoro- $N$-[4-[6-(isopropylamino)pyrimidin-4-yl]-1,3-thiazol-2-yl]- $N$ methylbenzamide $\left({ }^{18} \mathrm{~F}-\mathrm{FITM}\right)$ has been recently developed as a useful PET ligand for mGluR1 imaging in our laboratory. In this study, we aimed to measure the affinity and density of mGluR1 using PET with ${ }^{18} \mathrm{~F}$-FITM in rat brain under the in vivo conditions. Methods: Binding potentials $\left(\mathrm{BP}_{\mathrm{ND}}\right)$ and amounts of specific binding (bound ligand concentration) at equilibrium state in brain regions were noninvasively estimated using the equilibrium analysis combined with the receptor-blocked approach (EA RBA) for kinetic analysis of ${ }^{18} \mathrm{~F}$-FITM PET results in place of reference tissue methods. Using $\mathrm{BP}_{\mathrm{ND}}$ and specific binding values of rats treated with multidose ligand, we performed Scatchard analyses for in vivo measurements of mGluR1 density (maximum number of binding sites, or $\mathrm{B}_{\max }$ ) and ligand affinity (dissociation constant, or $\mathrm{K}_{\mathrm{d}}$ ) in brain regions, respectively. Results: The pretreatment of rats with unlabeled FITM (1 mg/kg) occupied an mGluR1 binding site of ${ }^{18} \mathrm{~F}$-FITM by more than $99 \%$ and did not affect the input function. Hence, we used the tissue time-activity curve for receptor-blocked rats as representative of the nondisplaceable (free and nonspecific binding of radioligand) compartment. The $\mathrm{BP}_{\mathrm{ND}}$ based on EA RBA showed a high correlation with the $\mathrm{BP}_{\mathrm{ND}}$ based on invasive Logan plot graphical analysis in the thalamus, hippocampus, striatum, and cingulate cortex. The $\mathrm{K}_{\mathrm{d}}(\mathrm{nM})$ and $\mathrm{B}_{\max }$ $(\mathrm{pmol} / \mathrm{mL})$ obtained by the Scatchard analyses with the multidose ligand assays were 2.1 and 36.3 , respectively, for the thalamus; 2.1 and 27.5, respectively, for the hippocampus; 1.5 and 22.2, respectively, for the striatum; and 1.5 and 20.5 , respectively, for the cingulate cortex with a high confidence. Conclusion: Our study is the first to our knowledge to measure the in vivo affinity $\left(K_{d}\right.$ and binding potential) of ${ }^{18} \mathrm{~F}-\mathrm{FITM}$ and mGluR1 density $\left(\mathrm{B}_{\max }\right)$ with a high correlation to in vitro values in rat brain regions. This measurement using PET with ${ }^{18} \mathrm{~F}-\mathrm{FITM}$ would be a useful index for research about mGluR1 functions in central nervous system disorders and development of new pharmaceuticals.

Received Mar. 13, 2012; revision accepted May 22, 2012.

For correspondence contact: Ming-Rong Zhang, Department of Molecular Probes, Molecular Imaging Center, National Institute of Radiological Sciences, 4-9-1, Anagawa, Inage-ku, Chiba 263-8555, Japan.

E-mail: zhang@nirs.go.jp

Published online Aug. 22, 2012.

COPYRIGHT @ 2012 by the Society of Nuclear Medicine and Molecular Imaging, Inc.
Key Words: ${ }^{18} \mathrm{~F}-\mathrm{FITM}$; mGluR1; $\mathrm{BP}_{\mathrm{ND}} ; \mathrm{K}_{\mathrm{d}}$; $\mathrm{B}_{\max }$

J Nucl Med 2012; 53:1601-1607

DOI: 10.2967/jnumed.112.105908

$\mathbf{T}$ he excitatory amino acid neurotransmitter glutamate is known to act via ionotropic receptors ( $\alpha$-amino-3-hydroxy5-methyl-4-isoxazolepropionic acid, $N$-methyl-D-aspartic acid, and kainite) and metabotropic receptors (metabotropic glutamate receptor subtypes 1-8 [mGluR1-8]) (1). MGluRs are members of the group $\mathrm{C}$ family of G-protein-coupled receptors and are divided into 3 different groups based on receptor sequence homology and physiologic activity. The mGluR1, along with mGluR5, is classified as group I (2). The activation of mGluR1 by endogenous glutamate stimulates intraneuronal cascades, which release $\mathrm{Ca}^{2+}$ from intracellular stores and activate protein kinase $\mathrm{C}$ (2). Thus, inhibition of mGluR1 activation has been indicated as potentially useful for neuroprotection against various injuries to the central nervous system, such as stroke (3-8), Parkinson disease (9-12), and Huntington disease (13).

Several radioligands for PET have been synthesized to investigate the in vivo localization and biologic characteristics of mGluR1 (14-17). Recently, we developed $4-{ }^{18}$ F-fluoro- $N$ [4-[6-(isopropylamino)pyrimidin-4-yl]-1,3-thiazol-2-yl]$N$-methylbenzamide $\left({ }^{18}\right.$ F-FITM) (Supplemental Fig. 1; supplemental materials are available online only at http:// jnm.snmjournals.org) (18) as a useful PET ligand for mGluR1 imaging in the brain.

PET is an advanced molecular imaging modality that enables the study of the living human brain and, in particular, specific proteins involved in pathophysiology or as targets for therapeutic interventions. In addition to its diagnostic applications, PET has been used to assess new therapeutic agents for targeting neuropsychiatric and neurodegenerative disorders in the brain (19). Tracking the dynamic biodistribution of radiolabeled compounds on maps of living animal brains has been made possible by the development of high-resolution PET scanners $(20,21)$. Thus, in vivo pharmacologic indices, 
such as the occupancy of the target binding sites by tested compounds and receptor density, can be commonly determined by PET with radioligands $(22,23)$. Moreover, multiple PET scans using living rodents allow high-accuracy data to be easily acquired, providing obvious advantages over ex vivo assays. Although mGluR1 is a crucial target molecule for neuropharmacology, in vivo measurement studies of mGluR1 in the brain using PET with a specific radioligand have never been performed.

In the present study, we measured the binding potential (BP), density (maximum number of binding sites, or $\mathrm{B}_{\max }$ ), and dissociation constant $\left(\mathrm{K}_{\mathrm{d}}\right)$ of mGluR1 in the rat brain using ${ }^{18}$ F-FITM PET studies.

\section{MATERIALS AND METHODS}

\section{General}

${ }^{18}$ F-FITM (Supplemental Fig. 1) was synthesized by ${ }^{18} \mathrm{~F}$ fluorination of a nitro precursor with ${ }^{18} \mathrm{~F}-\mathrm{KF} /$ Kryptofix 222 (Merck) as described previously (18). Unlabeled FITM was synthesized in our laboratory according to previously reported procedures (24). The compound was dissolved in saline containing $20 \%$ ethanol and $10 \%$ polyoxyethylene sorbitan monooleate (polysorbate 80 ) and used for in vivo studies.

Sprague-Dawley rats were purchased from Japan SLC and kept in a temperature-controlled environment with a 12-h light-dark cycle; the rats were fed a standard diet (MB-1; Funabashi Farm). Animal experiments were performed according to the recommendations of the Committee for the Care and Use of Laboratory Animals, National Institute of Radiological Sciences.

\section{Small-Animal PET Experiments}

PET Procedure. A Sprague-Dawley rat was secured in a customdesigned chamber and placed in a small-animal PET scanner (Inveon; Siemens Medical Solutions). Body temperature was maintained using a $40^{\circ} \mathrm{C}$ water circulation system (T/Pump TP401; Gaymar Industries). A 24-gauge intravenous catheter (Terumo Medical Products) was placed in the tail vein of the rat for a bolus injection.

A bolus of ${ }^{18}$ F-FITM (17-18 MBq, 30-40 pmol, $0.1 \mathrm{~mL}$ ) was injected via catheter in the tail vein of the rat, and dynamic emission scans in 3-dimensional list mode were obtained for $90 \mathrm{~min}$ (10 $\mathrm{s} \times 6$ scans, $15 \mathrm{~s} \times 4$ scans, $1 \mathrm{~min} \times 5$ scans, $2 \min \times 4$ scans, and $5 \min \times 15$ scans).

PET images were reconstructed using ASIPro VM (Analysis Tools and System Setup/Diagnostics Tool; Siemens Medical Solutions). Volumes of interest referencing the MRI template (25) were drawn on the cerebellum, thalamus, hippocampus, striatum, cingulate cortex, and pons. The sizes $\left(\mathrm{cm}^{2}\right)$ in each region of interest were 1.5 for the cerebellum, 1.2 for the thalamus, 0.3 for the hippocampus, 0.7 for the striatum, 0.2 for the cingulate cortex, and 0.5 for the pons. Each PET image was overlaid on the MRI template, and the time-activity curve for each region was characterized. The radioactivity was decay-corrected to the injection time and is expressed as the percentage of injected dose per milliliter $(\%$ $\mathrm{ID} / \mathrm{mL})$.

Arterial Blood Sampling. Before PET assessment, a SpragueDawley rat ( $n=4$; male; age, $8 \mathrm{wk}$; weight, 270-290 g) had a polyethylene catheter (FR2; Imamura) inserted into the left femoral artery for blood sampling. Arterial blood $(0.5-1 \mathrm{~mL})$ was manually sampled at 10, 20, 30, 40, and $50 \mathrm{~s}$ and 1, 2, 3, 4, 5, 10, 15, 30,
60, and 90 min after the PET scan started. The radioactivity in whole blood and plasma was counted using a 1480 Wizard Autogamma scintillation counter (Perkin-Elmer). Radioactivity was corrected for decay. Plasma treatment and metabolite analysis were performed according to a previous report (26). Similarly, the blocking study using rats (Sprague-Dawley; $n=4$; male; age, 8 wk; weight, 275$285 \mathrm{~g})$ pretreated with unlabeled FITM $(1 \mathrm{mg} / \mathrm{kg})$ was also performed as described above.

\section{Estimation of $\mathrm{BP}_{\mathrm{ND}}$ Using PET Kinetics}

Invasive Method. PET kinetic analyses were performed by Logan plot graphical analysis (GA) (27). The distribution volume $\left(\mathrm{V}_{\mathrm{T}}\right)$ of brain regions in the baseline and blocking studies was obtained using a plasma time-activity curve and tissue time-activity curve. All kinetic analyses were performed using PMOD software (version 3.2; PMOD Technologies).

To estimate nondisplaceable distribution volume $\left(\mathrm{V}_{\mathrm{ND}}\right)$, the Lassen plot GA was performed as described in the supplemental file.

The distribution volume of specific binding $\left(\mathrm{V}_{\mathrm{S}}\right)$ was obtained by subtracting the $\mathrm{V}_{\mathrm{ND}}$ based on the Lassen plot GA from the $\mathrm{V}_{\mathrm{T}}$ based on the Logan plot GA. The $\mathrm{BP}_{\mathrm{ND}}$, which equals the distribution volume ratio minus 1 , was determined according to the following equation:

$$
\begin{aligned}
\mathrm{BP}_{\mathrm{ND}}=\text { distribution volume ratio }-1=\frac{\mathrm{V}_{\mathrm{T}}-\mathrm{V}_{\mathrm{ND}}}{\mathrm{V}_{\mathrm{ND}}}=\frac{\mathrm{V}_{\mathrm{S}}}{\mathrm{V}_{\mathrm{ND}}} \text {. } \\
\text { Eq. } 1
\end{aligned}
$$

Noninvasive Method. The $\mathrm{BP}_{\mathrm{ND}}$ was noninvasively estimated to perform the Scatchard analysis with the multidose ligand assays. The equilibrium analysis (EA), in which saturated specific binding of the radioligand at equilibrium state is calculated from the specific binding time curve $\left(\mathrm{C}_{\mathrm{b}}\right)$ obtained by subtracting the tissue time-activity curve of the reference region $\left(\mathrm{C}_{\mathrm{ND}}\right)$ from the tissue time-activity curve in a region of interest $\left(\mathrm{C}_{\mathrm{T}}\right)$, has been previously introduced (28). In this analysis, the equilibrium state was defined as occurring at the time point when $\mathrm{dC}_{\mathrm{b}} / \mathrm{dt}$ for specific binding was 0 . The equilibrium time was defined by fitting to a $3-$ exponential equation. On the other hand, the receptor-blocked approach (RBA), in which the nondisplaceable compartment could be obtained by saturating a receptor with unlabeled ligand instead of using a reference tissue, has been previously reported (29). When a receptor binding site was significantly blocked by excess unlabeled ligand treatment, binding on-rate $\left(k_{3}\right)$ of the radioligand would be close to zero, indicating that its tissue time-activity curve was parallel to the nondisplaceable compartment. In this study, we combined EA with RBA because there was no reference region using PET with this radioligand. According to this approach, the $C_{b}$ in each brain region were determined by subtracting the tissue time-activity curve of the blocked rats as $\mathrm{C}_{\mathrm{ND}}$ from the tissue time-activity curve of the baseline rats as $\mathrm{C}_{\mathrm{T}}$, obtaining an equilibrium time using a 3-exponential fitting. The $\mathrm{BP}_{\mathrm{ND}}$ based on EA RBA was calculated according to the following equations:

$$
\begin{array}{cc}
\mathrm{C}_{\mathrm{b}}=\mathrm{C}_{\mathrm{T}}-\mathrm{C}_{\mathrm{ND}}, & \text { Eq. } 2 \\
\mathrm{BP}_{\mathrm{ND}}=\text { distribution volume ratio }-1=\frac{\mathrm{C}_{\mathrm{b}}^{(\mathrm{eq})}}{\mathrm{C}_{\mathrm{ND}}^{(\mathrm{eq})}}, & \text { Eq. } 3
\end{array}
$$

where $\mathrm{C}_{\mathrm{b}}^{(\mathrm{eq})}$ and $\mathrm{C}_{\mathrm{ND}}^{(\mathrm{eq})}$ express the specific binding and nondisplaceable binding concentrations $(\% \mathrm{ID} / \mathrm{mL})$ of tissue at equilibrium time. 
To validate $\mathrm{BP}_{\mathrm{ND}}$ based on the EA RBA, we generated a correlational scatterplot of $\mathrm{BP}_{\mathrm{ND}}$ based on the EA RBA against $\mathrm{BP}_{\mathrm{ND}}$ based on the Logan plot GA in each brain region.

\section{In Vivo Measurement of $B_{\max }$ and $K_{d}$ by ${ }^{18}$ F-FITM PET with Multidose Ligand Treatment}

The PET procedure was performed as described in the "SmallAnimal PET Experiments" section. Sprague-Dawley rats $(n=3$; male; age, 7-10 wk; weight, 220-310 g) were treated with different doses of unlabeled FITM $(0,1,5$, or $30 \mu \mathrm{g} / \mathrm{kg}$ or $1 \mathrm{mg} / \mathrm{kg})$ just before a bolus injection of ${ }^{18}$ F-FITM (17-18 MBq, 30-40 pmol, $0.1 \mathrm{~mL}$ ). Estimations of equilibrium state and $\mathrm{BP}_{\mathrm{ND}}$ were acquired in accordance with the approach as described in the "Estimation of $\mathrm{BP}_{\mathrm{ND}}$ Using PET Kinetics" section.

Scatchard analyses were performed to estimate the $B_{\max }$ and $K_{d}$ of mGluR1 with ${ }^{18}$ F-FITM. For the analysis, the amount of specific binding was plotted against $\mathrm{BP}_{\mathrm{ND}}$ instead of the ratio of bound ligand concentration to free ligand concentration under the in vitro condition. Values were calculated according to the following equations:

$$
\text { Specific binding }=\mathrm{C}_{\mathrm{b}} \times[\mathrm{L}] \text {, }
$$

$$
\begin{array}{r}
\frac{\text { Specific binding }}{\text { Free ligand concentration }} \times \mathrm{f}_{\mathrm{ND}}=\frac{\mathrm{C}_{\mathrm{b}}^{(\mathrm{eq})}}{\mathrm{C}_{\mathrm{ND}}^{(\mathrm{eq})}}=\mathrm{BP}_{\mathrm{ND}} \\
=\text { distribution volume ratio }-1,
\end{array}
$$

where $[\mathrm{L}]$ is the injected total ligand concentration $(\mathrm{pmol} / \mathrm{mL})$, and $f_{N D}$ represents the tissue free fraction in the nondisplaceable compartment. Note that $\mathrm{f}_{\mathrm{ND}}$ is close to $1.0(30)$.

Linear regression for the estimation of $B_{\max }$ and $K_{d}$ values for mGluR1 with ${ }^{18} \mathrm{~F}$-FITM in the thalamus, hippocampus, striatum, and cingulate cortex was performed using GraphPad Prism 5 (GraphPad Software). In this plot, $\mathrm{K}_{\mathrm{d}}$ is calculated as the multiplicative inverse of the slope of the regression line, and $\mathrm{B}_{\max }$ is determined from the $x$-axis intercept.

\section{RESULTS}

\section{Estimation of $\mathrm{BP}_{\mathrm{ND}}$ Using PET Kinetics}

Figure 1 shows the input functions (plasma time-activity curve) of ${ }^{18}$ F-FITM in baseline and blocked rat brains.

Plasma time-activity curves in both baseline and blocked rats showed initial uptake by $7 \%-8 \% \mathrm{ID} / \mathrm{mL}$ just after a bolus injection and rapid clearance by roughly $0.1 \% \mathrm{ID} / \mathrm{mL}$ at $15 \mathrm{~min}$. The pretreatment of rats with unlabeled FITM (1 mg/

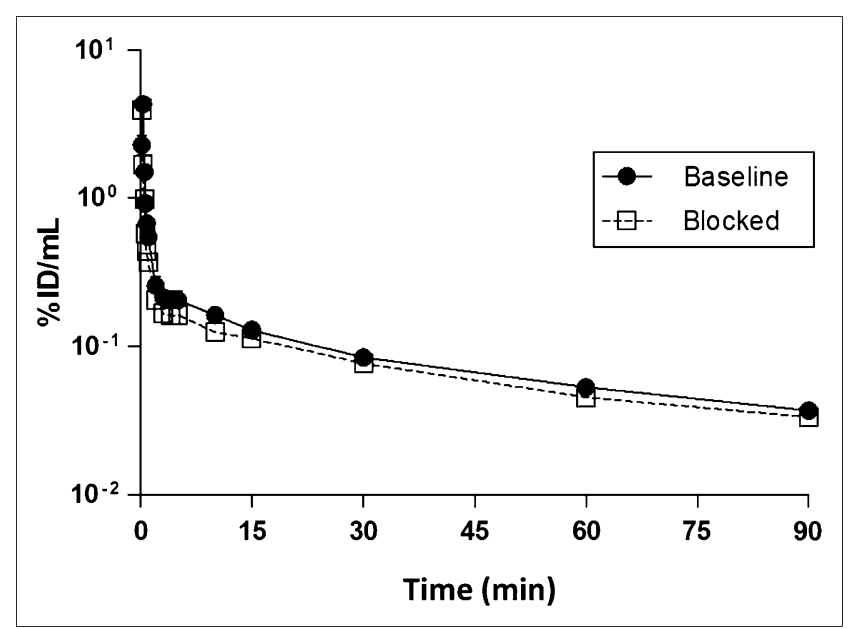

FIGURE 1. Time-activity curves of ${ }^{18} \mathrm{~F}$-FITM in plasma of baseline and blocked rats. Blocked rats were pretreated with unlabeled FITM $(1 \mathrm{mg} / \mathrm{kg}$ ). Plasma input function was corrected by injection time and metabolites of ${ }^{18} \mathrm{~F}-\mathrm{FITM}$. Mean \pm SE ( $n=4$ in each group).

$\mathrm{kg}$ ) barely influenced the input function. In the baseline tissue time-activity curves, remarkable accumulation of radioactivity was detected in the cerebellum; moderate uptake was detected in the thalamus, striatum, hippocampus, and cingulate cortex; and little uptake was found in the pons (Supplemental Fig. 2A). The pretreatment of rats with unlabeled FITM decreased uptake significantly and made it difficult to distinguish each region of the brain (Supplemental Fig. 2B).

The $\mathrm{V}_{\mathrm{T}}$ based on Logan plot GA in baseline and blocked rats is shown in Table 1 . In the baseline rats, the rank order of $\mathrm{V}_{\mathrm{S}}$ and $\mathrm{BP}_{\mathrm{ND}}$ using the $\mathrm{V}_{\mathrm{ND}}$ value based on the Lassen plot GA (Supplemental Fig. 3) among brain regions was cerebellum $>>$ thalamus $>$ striatum $>$ hippocampus $>$ cingulate cortex $>>$ pons (Table 1), corresponding to the reported mGluR1 densities in rat brain $(31)$.

Figure 2 shows the results of the EA RBA in the thalamus, hippocampus, striatum, and cingulate cortex.

The equilibrium time was $90 \mathrm{~min}$ for the thalamus, $76 \mathrm{~min}$ for the hippocampus, $74 \mathrm{~min}$ for the striatum, and 65 min for the cingulate cortex. $\mathrm{BP}_{\mathrm{ND}}$ according to Equation 3 was $18.4 \pm 1.9$ for the thalamus, $13.6 \pm 1.5$ for the hippocampus, $14.5 \pm 1.5$ for the striatum, and $13.0 \pm 1.4$ for the cingulate cortex.

TABLE 1

Results of Logan Plot GA in Brain Regions of Baseline and Blocked Rats

\begin{tabular}{lccrr}
\hline \multicolumn{1}{c}{ Region } & Baseline $\mathrm{V}_{\mathrm{T}}$ & Blocked $\mathrm{V}_{\mathrm{T}}$ & $\mathrm{V}_{\mathrm{S}}=\mathrm{V}_{\mathrm{T}}-\mathrm{V}_{\mathrm{ND}}{ }^{*}$ & $111.5 \pm 14.4$ \\
\hline Cerebellum & $114.0 \pm 14.4$ & $2.7 \pm 0.3$ & $56.5 \pm 5.8$ & $43.9 \pm 5.7$ \\
Thalamus & $59.1 \pm 5.8$ & $2.5 \pm 0.4$ & $45.4 \pm 4.5$ & $22.3 \pm 2.3$ \\
Hippocampus & $47.9 \pm 4.5$ & $2.4 \pm 0.4$ & $47.3 \pm 5.5$ & $17.9 \pm 1.8$ \\
Striatum & $49.9 \pm 5.5$ & $2.6 \pm 0.5$ & $39.4 \pm 4.3$ & $18.6 \pm 2.2$ \\
Cingulate cortex & $42.0 \pm 4.3$ & $2.4 \pm 0.3$ & $3.7 \pm 0.5$ & $15.5 \pm 1.7$ \\
Pons & $6.2 \pm 0.5$ & $2.7 \pm 0.4$ & $1.5 \pm 0.2$
\end{tabular}

*Value of $\mathrm{V}_{\mathrm{ND}}$ based on Lassen plot GA was $2.54 \mathrm{~mL} \cdot \mathrm{cm}^{-3}$ as shown in Supplemental Figure 3.

Data are mean $\pm \mathrm{SE}, n=4$. 
FIGURE 2. Identification of time point at which equilibrium occurs for mGluR1 with ${ }^{18} \mathrm{~F}-\mathrm{FITM}$ in thalamus (A), hippocampus (B), striatum (C), and cingulate cortex (D). Measured values in baseline experiments were subtracted from measured values in blocked experiments. Difference was defined as specific binding and fitted to set of 3 exponentials. Each equilibrium time is indicated by black arrow. Points are expressed as mean \pm SE $(n=4)$.

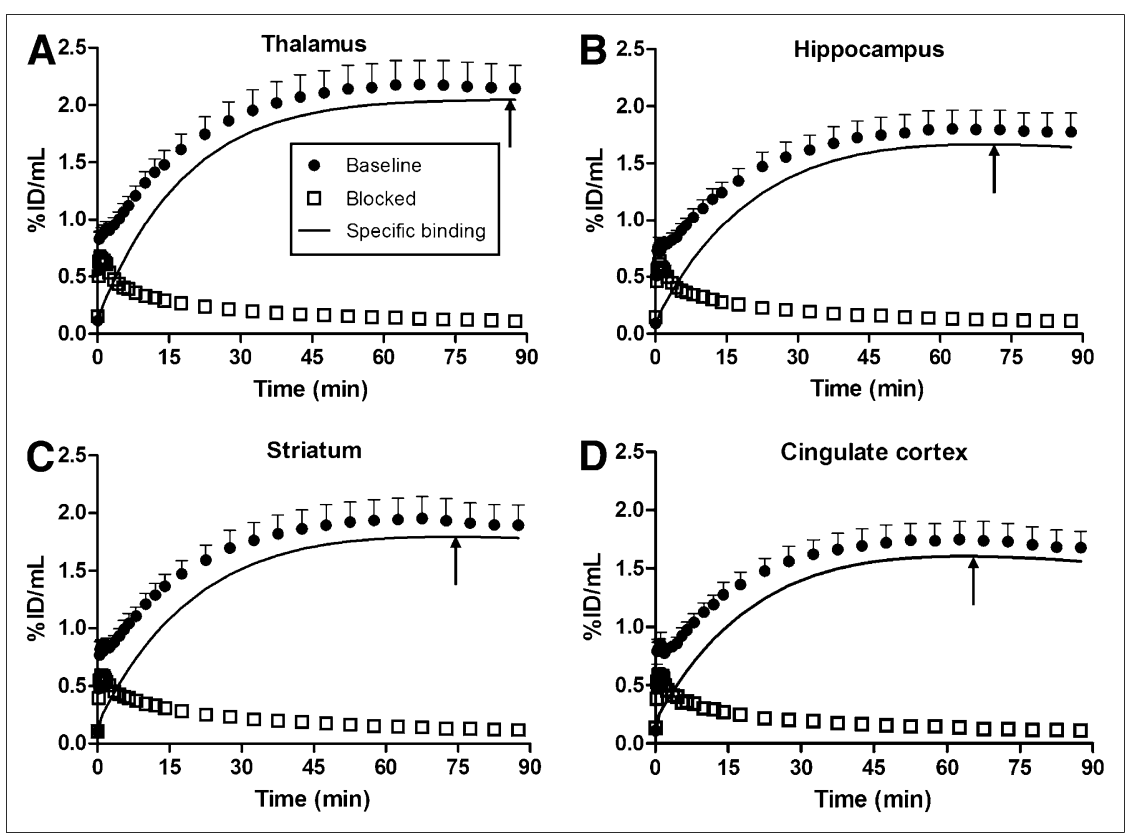

Figure 3 shows the results of linear regression using plots of $\mathrm{BP}_{\mathrm{ND}}$ based on the EA RBA against $\mathrm{BP}_{\mathrm{ND}}$ based on the Logan plot GA. The regression line had good confidence $\left(R^{2}=0.93\right)$, and the slope was 0.90. Although the $\mathrm{BP}_{\mathrm{ND}}$ based on EA RBA showed a result of roughly $10 \%$ underestimations, compared with that based on Logan plot GA, the good linear regression result supported the use of the EA RBA instead of reference tissue methods. The EA RBA

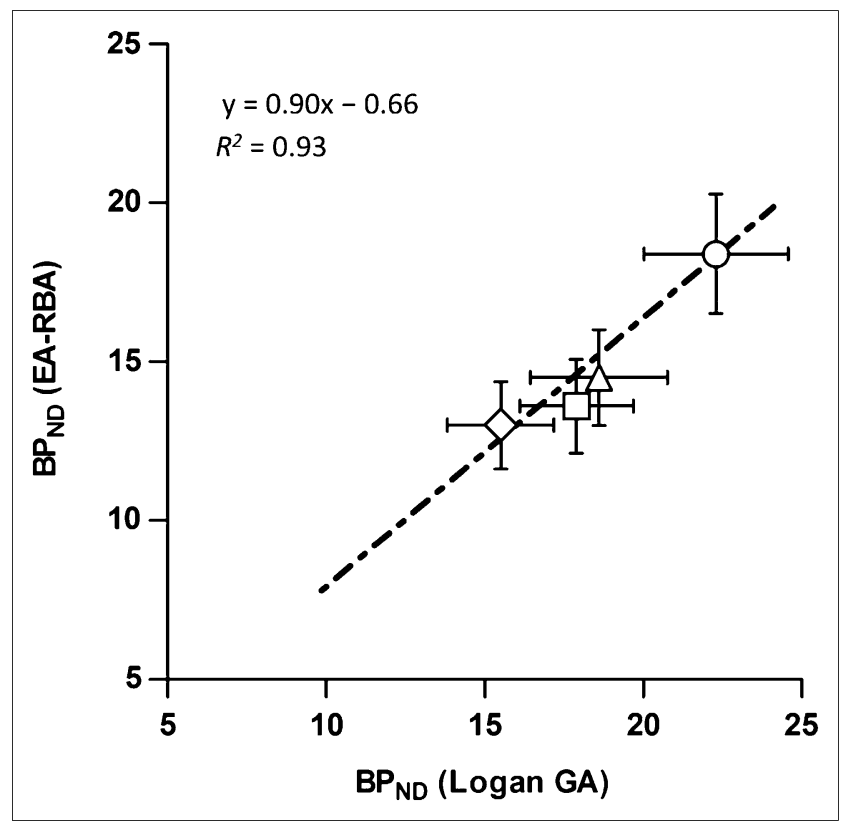

FIGURE 3. Correlational scatterplots between $\mathrm{BP}_{\mathrm{ND}}$ values based on EA RBA and those based on Logan plot GA. Plots show average datum points, with SEs in $x$ - and $y$-axes $(n=4)$. $\bigcirc=$ thalamus; $\square=$ hippocampus; $\triangle$ = striatum; $\diamond=$ cingulate cortex. could not be used in the cerebellum and pons because of the poor regression in those regions.

\section{In Vivo Measurement of $B_{\max }$ and $K_{d}$ by ${ }^{18}$ F-FITM PET with Multidose Ligand Treatment}

Figure 4 shows representative PET images of the thalamus/ hippocampus, striatum, and cingulate cortex on the coronal slices in baseline rats and rats pretreated with different doses of unlabeled FITM $(1,5$, and $30 \mu \mathrm{g} / \mathrm{kg}$ and $1 \mathrm{mg} / \mathrm{kg}$, Figs. $4 \mathrm{~B}, 4 \mathrm{C}, 4 \mathrm{D}$, and $4 \mathrm{E}$, respectively). With increasing doses of unlabeled FITM, the radioactivity showed a stepwise attenuation in all regions.

The dose-dependent reduction of ${ }^{18} \mathrm{~F}$-FITM binding to mGluR1 in the multidose ligand assays was shown by alterations to the time curve of specific binding in the thalamus, hippocampus, striatum, and cingulate cortex (Supplemental Fig. 4). The transient equilibrium of specific binding $\left(\mathrm{dC}_{\mathrm{b}} /\right.$ $\mathrm{dt}=0$ ) based on EA RBA was reached at 70-90 $\mathrm{min}$ in the thalamus, $55-75 \mathrm{~min}$ in the hippocampus, $60-80 \mathrm{~min}$ in the striatum, and 50-70 $\mathrm{min}$ in the cingulate cortex (Supplemental Fig. 4).

Figure 5 shows the Scatchard plots for the thalamus, hippocampus, striatum, and cingulate cortex. Table 2 shows $\mathrm{B}_{\max }, \mathrm{K}_{\mathrm{d}}$, the coefficient of determination $\left(R^{2}\right)$, and $\mathrm{BP}$ $\left(B_{\max } / K_{d}\right)$ values in each brain region. The $K_{d}$ values in all brain regions were in the range of 1.5-2.1, with good confidence of linear fit $\left(R^{2}=0.87-0.96\right)$.

\section{DISCUSSION}

To our knowledge, the present ${ }^{18}$ F-FITM PET studies provided in vivo measurements of $B_{\max }$ and $K_{d}$ in rat brain for the first time.

We have developed ${ }^{18}$ F-FITM as a novel radioligand of mGluR1 imaging and demonstrated its potential in in vitro and in vivo studies of mGluR1 in rodent and nonhuman 

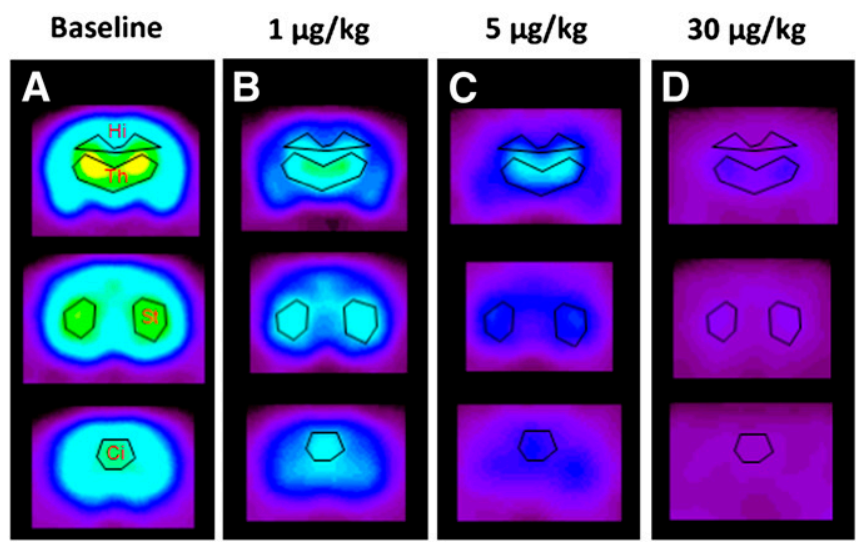

\section{$1 \mathrm{mg} / \mathrm{kg}$}

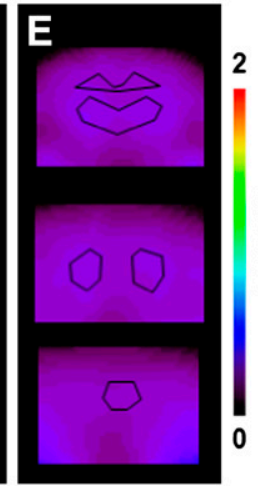

FIGURE 4. PET of ${ }^{18} \mathrm{~F}-\mathrm{FITM}$ distribution in rat brains after intravenous injection of unlabeled FITM. Rats were pretreated with different doses of unlabeled FITM: baseline (A), $1 \mu \mathrm{g} / \mathrm{kg}$ (B), $5 \mu \mathrm{g} / \mathrm{kg}$ (C), $30 \mu \mathrm{g} / \mathrm{kg}$ (D), and $1 \mathrm{mg} / \mathrm{kg}$ (E) administered). Coronal PET images were generated by averaging $d y-$ namic data at 0-90 min after bolus injection of ${ }^{18} \mathrm{~F}$-FITM. Volumes of interest were placed on hippocampus, thalamus, striatum, and cingulate cortex. $\mathrm{Hi}=$ hippocampus; $\mathrm{Th}=$ thalamus; $\mathrm{St}=$ striatum; $\mathrm{Ci}=$ cingulate cortex.

primate brains $(18,32)$. In those studies, we confirmed the pharmacologic selectivity of ${ }^{18}$ F-FITM on the basis of competition with a mGluR1-selective ligand such as JNJ16259685. In a preliminary experiment, we examined brain uptake of ${ }^{18} \mathrm{~F}-$ FITM in wild-type and mGluR1 knockout mice. Brain uptake of radioactivity was barely detected in the PET image of the mGluR1 knockout mouse (Supplemental Fig. 5). These results directly demonstrate the high specificity of ${ }^{18}$ F-FITM for mGluR1, indicating that this radioligand is a promising tracer suitable for the accurate in vivo measurement of pharmacologic indices for mGluR1 in the brain.

$\mathrm{BP}$, defined as the ratio of bound ligand concentration to free ligand concentration, was introduced in the earliest

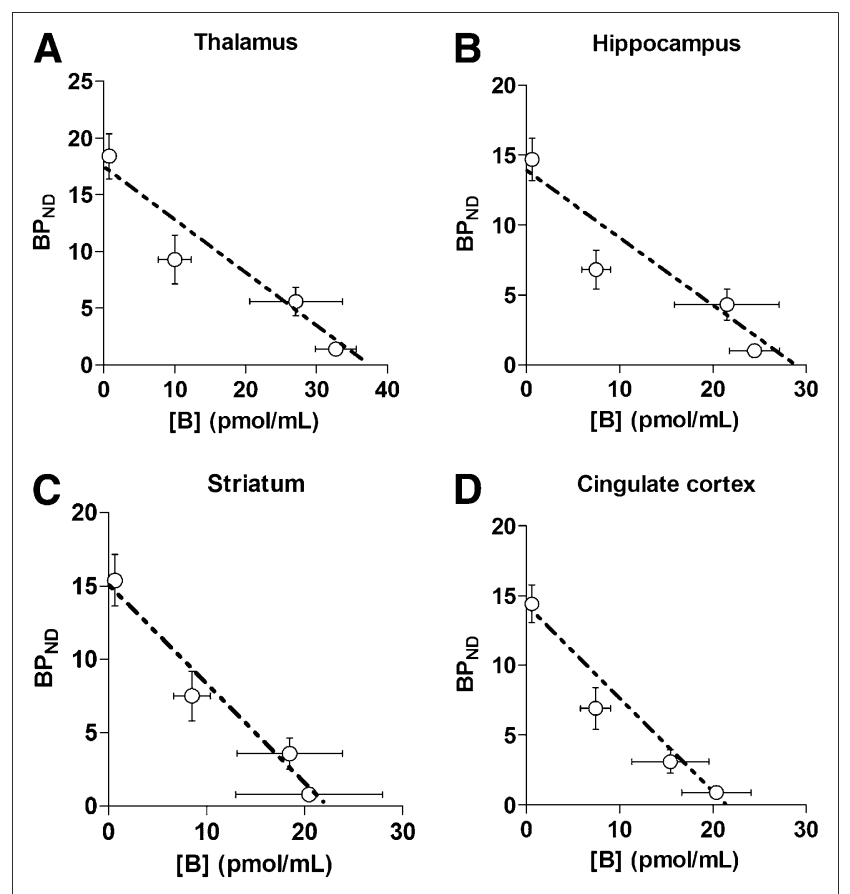

FIGURE 5. Scatchard analyses for determination of mGluR1 $B_{\max }$ and $\mathrm{K}_{\mathrm{d}}$ of ${ }^{18} \mathrm{~F}-\mathrm{FITM}$ in thalamus (A), hippocampus (B), striatum (C), and cingulate cortex (D). Regression lines are indicated for each region. The plots were expressed by average datum points, with standard errors in $x$ - and $y$-axis $(n=3)$. $[\mathrm{B}]=$ bound ligand concentration. days of PET application to the study of neuroreceptors in the brain (33). Scatchard analysis has often been performed for the in vivo determination of $\mathrm{B}_{\max }$ and $\mathrm{K}_{\mathrm{d}}$ using $\mathrm{BP}$ values and the amount of specific binding of the ligand (34-36). In general, the BP, under typical assumptions, can be directly calculated only from brain data using a variety of reference tissue methods such as $\mathrm{BP}_{\mathrm{ND}}$ in vivo.

The brain stem is known to have negligible expression of mGluR 1 by immunohistochemistry (31) and autoradiograms with several radioligands including ${ }^{18} \mathrm{~F}$-FITM $(14,15,17,18,37)$. Therefore, we assumed that the pons, a part of the brain stem, could be used as a reference region. To verify the aptitude of the pons, we measured the $\mathrm{V}_{\mathrm{T}}$ of rat brain regions by Logan plot GA in baseline and blocking studies. Unfortunately, apparent specific binding in the pons was detected; the $\mathrm{V}_{\mathrm{T}}$ values in baseline and blocking studies were 6.2 and 2.7, respectively (Table 1). Thus, the reference region methods were not adaptable to in vivo measurement for pharmacologic indices of this radioligand because of an underestimation of $\mathrm{BP}_{\mathrm{ND}}$. In fact, the $\mathrm{BP}_{\mathrm{ND}}$ based on the simplified reference tissue model (38) using the tissue timeactivity curve of the pons was underestimated by roughly $40 \%-50 \%$ in all brain regions, compared with the $\mathrm{BP}_{\mathrm{ND}}$ (distribution volume ratio - 1) based on a 2-tissue-compartment model.

To noninvasively measure $\mathrm{BP}_{\mathrm{ND}}$, we used the RBA, which was able to estimate the distribution volume of the free and nonspecific binding compartment, instead of using the reference tissue (29). However, with this approach, there is a potential concern that the input function may be changed by treatment with excess unlabeled ligand and that the injected unlabeled ligand dose may not fully occupy a binding site of the receptor. In the present study, the plasma time-activity curve of ${ }^{18}$ F-FITM with blockade of specific binding did not show an acute increase because of overflow from the peripherals at the initial phase (Fig. 1). Although the initial peak in the plasma time-activity curve in the blocked rats occurred earlier than that in the baseline rats, this potential concern is unnecessary because all tissue time-activity curves peaked more than $45 \mathrm{~min}$ after the ${ }^{18} \mathrm{~F}$ FITM injection (Supplemental Fig. 2A). Another concern is 
TABLE 2

In Vivo Measurements of $K_{d}, B_{\max }$, and BP by Scatchard Analyses in Rat Brain

\begin{tabular}{lcccc}
\hline \multicolumn{1}{c}{ Region } & $\begin{array}{c}\mathrm{K}_{\mathrm{d}} \\
(\mathrm{nM})\end{array}$ & $\begin{array}{c}\mathrm{B}_{\max } \\
(\mathrm{pmol} / \mathrm{mL})\end{array}$ & $R^{2 *}$ & $\mathrm{BP}^{\dagger}$ \\
\hline Thalamus & 2.1 & 36.3 & 0.91 & 17.3 \\
Hippocampus & 2.1 & 27.5 & 0.87 & 13.1 \\
Striatum & 1.5 & 22.2 & 0.96 & 14.8 \\
Cingulate cortex & 1.5 & 20.5 & 0.95 & 13.7 \\
& & & & \\
\hline${ }^{*}$ Coefficient of determination. & & & \\
${ }^{\dagger} \mathrm{BP}=\mathrm{B}_{\text {max }} / \mathrm{K}_{\mathrm{d}}$. & & & \\
\hline
\end{tabular}

the ligand occupancy of the targeted receptor. As shown in the tissue time-activity curves of the blocked rats (Supplemental Fig. 2B), the uptake was significantly lower than that in baseline tissue time-activity curves in each brain region measured. The ligand occupancy and $\mathrm{V}_{\mathrm{ND}}$ were calculated using Lassen plot GA with linear regression (Supplemental Fig. 3). It was shown that pretreatment with $1 \mathrm{mg}$ of unlabeled FITM per kilogram occupied mGluR1 binding sites at more than $99 \%$ in each brain region. This regression analysis showed that the tissue time-activity curve for each brain region in the blocked rats was almost equal to the nondisplaceable compartment, which can be used instead of reference tissue.

To perform the Scatchard analysis with the multidose ligand assays, we estimated $\mathrm{BP}_{\mathrm{ND}}$ based on the EA RBA in rat brain regions. Specific binding time curves were obtained by EA (Fig. 2) (28). For validation of the EA RBA, we generated a correlational scatterplot between $\mathrm{BP}_{\mathrm{ND}}$ based on the EA RBA and that based on the Logan plot GA (Fig. 3). The regression line showed good correlation in the thalamus, hippocampus, striatum, and cingulate cortex. However, plots of the cerebellum, a region with abundant mGluR1, and the pons, a region with negligible mGluR1, were removed from the regression line because of their nonadaptability to the EA RBA. In the cerebellum, the equilibrium state of ${ }^{18} \mathrm{~F}-$ FITM has been previously shown to be over $180 \mathrm{~min}$ (32). The in vivo measurements of $B_{\max }$ and $K_{d}$ of mGluR1 with ${ }^{18}$ F-FITM in the cerebellum were not suited to the EA RBA, because the EA could be applied in vivo only when a specific binding reached a peak within the time span of a PET experiment (28). More importantly, PET with this radioligand was suited to quantification of mGluR1 in moderate- or lowdensity regions, although the in vivo quantification of mGluR1 with ${ }^{18}$ F-FITM in the cerebellum would be inferior to that with ${ }^{18} \mathrm{~F}-\mathrm{MK}-1312$ (16), a promising PET ligand for cerebellar mGluR1. ${ }^{18} \mathrm{~F}-\mathrm{MK}-1312$ PET analysis with a 2-tissue-compartment model in a monkey showed good kinetic parameters with high confidence in the cerebellum, but the directly estimated $\mathrm{BP}_{\mathrm{ND}}\left(k_{3} / k_{4}\right)$ was under 1.0. In addition, significant specific binding of ${ }^{18} \mathrm{~F}-\mathrm{MK}-$ 1312 was detected in the cerebellum but not in the thalamus and striatum.
On the other hand, the pons, a region with negligible mGluR1, is located near the cerebellum, the region with the highest accumulation of radioactivity. Therefore, nonsaturated radioactive signals in the cerebellum would spill into the pons. This profile might be the cause of the nonadaptability of reference tissue models to the pons. However, the rat brain is considerably smaller than the human brain, and partial-volume errors cause spill-in of radioactivity from adjacent areas. The larger human brain may have regions with no (or limited) displaceable uptake that could hypothetically be used as a reference region. Although ${ }^{18}$ F-FITM with EA RBA was not useful in the cerebellum and pons, which in the moderate- or low-mGluR1 brain regions-such as the thalamus, hippocampus, striatum, and cingulate cortexdemonstrated the ability to noninvasively quantify mGluR1.

Interestingly, the expression levels of mGluR1 in animal models of central nervous system disorders, such as Parkinson disease and Alzheimer disease, are altered in the thalamus, striatum, and cerebral cortex but not in the cerebellum $(9,39,40)$. To measure $B_{\max }$ and $K_{d}$ values in vivo in the thalamus, hippocampus, striatum, and cingulate cortex, we performed Scatchard analyses with multidose ligand assays using the $\mathrm{BP}_{\mathrm{ND}}$ and the amount (pmol/mL) of specific binding based on the EA RBA (Fig. 5). As shown in Table 2, $K_{d}$ and $B_{\max }$ values in each region were calculated from a regression line with high correlation $\left(R^{2}=0.87-0.96\right)$.

We previously measured the in vitro $\mathrm{K}_{\mathrm{d}}$ value $(1.9 \mathrm{nM})$ of ${ }^{18}$ F-FITM using a rat brain homogenate (37). Theoretically, the $K_{d}$ value is a fundamentally impartial value in both in vitro and in vivo conditions. However, in vivo measurements are sometimes different from in vitro measurements because of the metabolism of the radioligand, high amounts of nonspecific binding, the influence of transporters, and a variety of other reasons. In the present study, the in vivo $\mathrm{K}_{\mathrm{d}}$ values in the thalamus, hippocampus, striatum, and cingulate cortex were all in the range of 1.5-2.1, approximating the in vitro measured value. These results suggested that PET with ${ }^{18} \mathrm{~F}-$ FITM is not affected by in vivo factors. The EA RBA with ${ }^{18} \mathrm{~F}-\mathrm{FITM}$ demonstrated relatively accurate measurement of mGluR1 density ( $\mathrm{pmol} / \mathrm{mL})$ in the diencephalon and telencephalon, such as 36.3 for the thalamus, 27.5 for the hippocampus, 22.2 for the striatum, and 20.5 for the cingulate cortex.

\section{CONCLUSION}

We have determined the $B_{\max }$ and $K_{d}$ of mGluR1 in the thalamus, hippocampus, striatum, and cingulate cortex using ${ }^{18}$ F-FITM PET combined with the EA RBA using multidose ligand assays. ${ }^{18} \mathrm{~F}$-FITM PET is a promising tool for the further understanding of the mGluR1 function under pathophysiologic conditions. The present results will aid the development of pharmaceuticals targeting central nervous system disorders, for which it is important to measure indices such as mGluR1 occupancy and to determine effective dose in a target brain region. 


\section{DISCLOSURE STATEMENT}

The costs of publication of this article were defrayed in part by the payment of page charges. Therefore, and solely to indicate this fact, this article is hereby marked "advertisement" in accordance with 18 USC section 1734.

\section{ACKNOWLEDGMENTS}

We thank Tatsumi Hirata (National Institute of Genetics, Shizuoka, Japan) for development of the mGluR1 knockout mouse. We are also grateful to the staff of the National Institute of Radiological Sciences for assistance with the cyclotron operation, radiosynthesis, and animal experiments. This work was partly supported by a Grant-in-Aid for Basic Research (C) (22591379) from the Ministry of Education, Culture, Sports, Science and Technology of the Japanese Government. No other potential conflict of interest relevant to this article was reported.

\section{REFERENCES}

1. Kew JN, Kemp JA. Ionotropic and metabotropic glutamate receptor structure and pharmacology. Psychopharmacology (Berl). 2005;179:4-29.

2. Ferraguti F, Crepaldi L, Nicoletti F. Metabotropic glutamate 1 receptor: current concepts and perspectives. Pharmacol Rev. 2008;60:536-581.

3. Zhou M, Xu W, Liao G, Bi X, Baudry M. Neuroprotection against neonatal hypoxia/ischemia-induced cerebral cell death by prevention of calpain-mediated mGluR1alpha truncation. Exp Neurol. 2009;218:75-82.

4. De Vry J, Horvath E, Schreiber R. Neuroprotective and behavioral effects of the selective metabotropic glutamate $\mathrm{mGlu}_{1}$ receptor antagonist BAY 36-7620. Eur J Pharmacol. 2001;428:203-214

5. Kohara A, Takahashi M, Yatsugi S, et al. Neuroprotective effects of the selective type 1 metabotropic glutamate receptor antagonist YM-202074 in rat stroke models. Brain Res. 2008;1191:168-179

6. Moroni F, Attucci S, Cozzi A, et al. The novel and systemically active metabotropic glutamate 1 (mGlu1) receptor antagonist 3-MATIDA reduces post-ischemic neuronal death. Neuropharmacology. 2002;42:741-751.

7. Murotomi K, Takagi N, Takayanagi G, Ono M, Takeo S, Tanonaka K. mGluR1 antagonist decreases tyrosine phosphorylation of NMDA receptor and attenuates infarct size after transient focal cerebral ischemia. J Neurochem. 2008;105:16251634.

8. Szydlowska K, Kaminska B, Baude A, Parsons CG, Danysz W. Neuroprotective activity of selective mGlu1 and mGlu5 antagonists in vitro and in vivo. Eur $J$ Pharmacol. 2007;554:18-29.

9. Kaneda K, Tachibana Y, Imanishi M, et al. Down-regulation of metabotropic glutamate receptor 1alpha in globus pallidus and substantia nigra of parkinsonian monkeys. Eur J Neurosci. 2005;22:3241-3254.

10. Dekundy A, Pietraszek M, Schaefer D, Cenci MA, Danysz W. Effects of group I metabotropic glutamate receptors blockade in experimental models of Parkinson's disease. Brain Res Bull. 2006;69:318-326.

11. Ossowska K, Wardas J, Pietraszek M, Konieczny J, Wolfarth S. The striopallidal pathway is involved in antiparkinsonian-like effects of the blockade of group I metabotropic glutamate receptors in rats. Neurosci Lett. 2003;342:21-24.

12. Vernon AC, Croucher MJ, Dexter DT. Additive neuroprotection by metabotropic glutamate receptor subtype-selective ligands in a rat Parkinson's model. Neuroreport. 2008;19:475-478.

13. Ribeiro FM, Paquet M, Ferreira LT, et al. Metabotropic glutamate receptormediated cell signaling pathways are altered in a mouse model of Huntington's disease. J Neurosci. 2010;30:316-324.

14. Fujinaga M, Maeda J, Yui J, et al. Characterization of 1-(2-[ $\left.{ }^{18} \mathrm{~F}\right]$ fluoro-3-pyridyl)4-(2-isopropyl-1-oxo- isoindoline-5-yl)-5-methyl-1H-1,2,3-triazole, a PET ligand for imaging the metabotropic glutamate receptor type 1 in rat and monkey brains. J Neurochem. 2012;121:115-124.

15. Fujinaga M, Yamasaki T, Kawamura K, et al. Synthesis and evaluation of 6-[1(2- $\left[{ }^{18} \mathrm{~F}\right]$ fluoro-3-pyridyl)-5-methyl-1H-1,2,3-triazol-4-yl]quinoline for positron emission tomography imaging of the metabotropic glutamate receptor type 1 in brain. Bioorg Med Chem. 2011;19:102-110.
16. Hostetler ED, Eng W, Joshi AD, et al. Synthesis, characterization, and monkey PET studies of $\left[{ }^{18} \mathrm{~F}\right] \mathrm{MK}-1312$, a PET tracer for quantification of mGluR1 receptor occupancy by MK-5435. Synapse. 2011;65:125-135.

17. Yanamoto K, Konno F, Odawara C, et al. Radiosynthesis and evaluation of $\left[{ }^{11} \mathrm{C}\right]$ YM-202074 as a PET ligand for imaging the metabotropic glutamate receptor type 1. Nucl Med Biol. 2010;37:615-624.

18. Yamasaki T, Fujinaga M, Yoshida Y, et al. Radiosynthesis and preliminary evaluation of $4-\left[{ }^{18} \mathrm{~F}\right]$ fluoro- $N$-[4-[6-(isopropylamino)pyrimidin-4-yl]-1,3-thiazol-2yl]-N- methylbenzamide as a new positron emission tomography ligand for metabotropic glutamate receptor subtype 1. Bioorg Med Chem Lett. 2011;21: 2998-3001.

19. Saijo T, Maeda J, Okauchi T, et al. Utility of small-animal positron emission tomographic imaging of rats for preclinical development of drugs acting on the serotonin transporter. Int J Neuropsychopharmacol. 2009;12:1021-1032.

20. Tai YC, Ruangma A, Rowland D, et al. Performance evaluation of the microPET focus: a third-generation microPET scanner dedicated to animal imaging. $\mathrm{J} \mathrm{Nucl}$ Med. 2005;46:455-463.

21. Yang Y, Tai YC, Siegel S, et al. Optimization and performance evaluation of the microPET II scanner for in vivo small-animal imaging. Phys Med Biol. 2004;49: 2527-2545.

22. Fowler JS, Volkow ND, Wang GJ, Ding YS, Dewey SL. PET and drug research and development. J Nucl Med. 1999;40:1154-1163.

23. Wang J, Maurer L. Positron emission tomography: applications in drug discovery and drug development. Curr Top Med Chem. 2005;5:1053-1075.

24. Satoh A, Nagatomi Y, Hirata Y, et al. Discovery and in vitro and in vivo profiles of 4-fluoro- $N$-[4-[6-(isopropylamino)pyrimidin-4-yl]-1,3-thiazol-2-yl]-N-methy lbenzamide as novel class of an orally active metabotropic glutamate receptor 1 (mGluR1) antagonist. Bioorg Med Chem Lett. 2009;19:5464-5468.

25. Yui J, Maeda J, Kumata K, et al. ${ }^{18}$ F-FEAC and ${ }^{18}$ F-FEDAC: PET of the monkey brain and imaging of translocator protein $(18 \mathrm{kDa})$ in the infarcted rat brain. J Nucl Med. 2010;51:1301-1309.

26. Kawamura K, Naganawa M, Konno F, et al. Imaging of I2-imidazoline receptors by small-animal PET using 2-(3-fluoro-[4-11 C]tolyl)-4,5-dihydro- $1 H$-imidazole ([ $\left.{ }^{11} \mathrm{C}\right]$ FTIMD). Nucl Med Biol. 2010;37:625-635.

27. Logan J, Fowler JS, Volkow ND, et al. Graphical analysis of reversible radioligand binding from time-activity measurements applied to $\left[N-{ }^{11} \mathrm{C}\right.$-methyl $]-(-)$-cocaine PET studies in human subjects. J Cereb Blood Flow Metab. 1990;10:740-747.

28. Farde L, Eriksson L, Blomquist G, Halldin C. Kinetic analysis of central $\left[{ }^{11} \mathrm{C}\right]$ raclopride binding to D2-dopamine receptors studied by PET-a comparison to the equilibrium analysis. J Cereb Blood Flow Metab. 1989;9:696-708.

29. Sakiyama Y, Saito M, Inoue O. Acute treatment with pentobarbital alters the kinetics of in vivo receptor binding in the mouse brain. Nucl Med Biol. 2006;33:535-541.

30. Innis RB, Cunningham VJ, Delforge J, et al. Consensus nomenclature for in vivo imaging of reversibly binding radioligands. J Cereb Blood Flow Metab. 2007;27: 1533-1539.

31. Fotuhi M, Sharp AH, Glatt CE, et al. Differential localization of phosphoinositidelinked metabotropic glutamate receptor (mGluR1) and the inositol 1,4,5trisphosphate receptor in rat brain. J Neurosci. 1993;13:2001-2012.

32. Yamasaki T, Fujinaga M, Maeda J, et al. Imaging for metabotropic glutamate receptor subtype 1 in rat and monkey brains using PET with $\left[{ }^{18}\right.$ F]FITM. Eur $J$ Nucl Med Mol Imaging. 2012;39:632-641.

33. Mintun MA, Raichle ME, Kilbourn MR, Wooten GF, Welch MJ. A quantitative model for the in vivo assessment of drug binding sites with positron emission tomography. Ann Neurol. 1984;15:217-227.

34. Doudet DJ, Jivan S, Ruth TJ, Holden JE. Density and affinity of the dopamine D2 receptors in aged symptomatic and asymptomatic MPTP-treated monkeys: PET studies with $\left[{ }^{11}\right.$ C $]$ raclopride. Synapse. 2002;44:198-202.

35. Fischer K, Sossi V, Schmid A, et al. Noninvasive nuclear imaging enables the in vivo quantification of striatal dopamine receptor expression and raclopride affinity in mice. J Nucl Med. 2011;52:1133-1141.

36. Holden JE, Jivan S, Ruth TJ, Doudet DJ. In vivo receptor assay with multiple ligand concentrations: an equilibrium approach. J Cereb Blood Flow Metab. 2002;22:1132-1141.

37. Fujinaga M, Yamasaki T, Yui J, et al. Synthesis and evaluation of novel radioligands for positron emission tomography imaging of metabotropic glutamate receptor subtype 1 (mGluR1) in the brain. J Med Chem. 2012;55:2342-2352.

38. Lammertsma AA, Hume SP. Simplified reference tissue model for PET receptor studies. Neuroimage. 1996;4:153-158.

39. Kaneda K, Imanishi M, Nambu A, Shigemoto R, Takada M. Differential expression patterns of mGluR1 alpha in monkey nigral dopamine neurons. Neuroreport. 2003;14:947-950.

40. Roselli F, Hutzler P, Wegerich Y, Livrea P, Almeida OF. Disassembly of shank and homer synaptic clusters is driven by soluble beta-amyloid(1-40) through divergent NMDAR-dependent signalling pathways. PLoS ONE. 2009;4:e6011. 